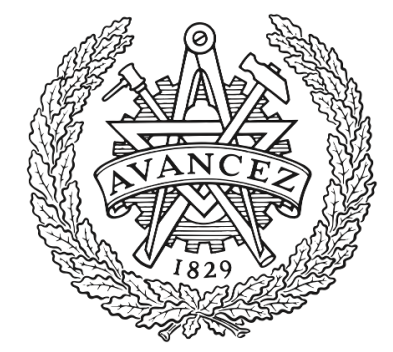

CHALMERS

UNIVERSITY OF TECHNOLOGY

\title{
A Broadband, Low Noise, Integrated 340 GHz Schottky Diode Receiver
}

Downloaded from: https://research.chalmers.se, 2023-04-26 11:57 UTC

Citation for the original published paper (version of record):

Sobis, P., Wadefalk, N., Emrich, A. et al (2012). A Broadband, Low Noise, Integrated $340 \mathrm{GHz}$ Schottky Diode Receiver. IEEE Microwave and Wireless Components Letters, 22(7): 366-368. http://dx.doi.org/10.1109/LMWC.2012.2202280

N.B. When citing this work, cite the original published paper.

(O2012 IEEE. Personal use of this material is permitted.

However, permission to reprint/republish this material for advertising or promotional purposes 


\title{
A Broadband, Low Noise, Integrated $340 \mathrm{GHz}$ Schottky Diode Receiver
}

\author{
Peter J. Sobis, Student Member, IEEE, Niklas Wadefalk, Anders Emrich, and Jan Stake, Senior \\ Member, IEEE
}

\begin{abstract}
A 340 GHz subharmonic Schottky diode mixer and a multioctave (3-17 GHz) custom LNA have been integrated to form a compact receiver front-end module, exhibiting ultra low noise with an exceptional flat response and broadband instantaneous frequency coverage. At room temperature, a receiver noise temperature of $870 \mathrm{~K}$ is measured at an LO drive of $1.2 \mathrm{~mW}$ at $170 \mathrm{GHz}$. The total DC power consumption of the LNA is below $120 \mathrm{~mW}$. Measurements are in good agreement with simulations taking the mixer and LNA mismatch interaction into account.
\end{abstract}

Index Terms-Submillimeter wave technology, Schottky diodes, heterodyne receivers, radiometers, subharmonic mixers, low noise amplifiers

\section{INTRODUCTION}

$\mathrm{T}$ HE next generation Earth science missions and planetary exploration missions $[1,2]$ will rely on broadband state-ofthe-art submillimeter wave heterodyne Schottky receivers. The low weight, high reliability and long operational lifetime, compared to cryogenic receiver systems [3], are the main qualities that make room temperature technology suitable for this type of applications.

The core elements of a Schottky receiver front-end are the mixer [4], most efficiently realized as a subharmonic mixer, and the IF low noise amplifier LNA [5]. In general the integration and co-optimization of critical components in the front-end receiver system can improve performance considerably, increasing bandwidth, reducing power consumption and size etc. [6-9]. For heterodyne Schottky

Manuscript received March 2012. This work was carried out in the GigaHertz Centre in a joint project in part financed by Swedish Governmental Agency of Innovation Systems (VINNOVA), Chalmers University of Technology, Wasa Millimeter Wave AB, Omnisys Instruments AB and SP Technical Research Institute of Sweden. The work was also supported in part by the Swedish Research Council (VR) under grant no. 2005-2855.

P. J. Sobis is with Omnisys Instruments AB and with the Gigahertz Centre, Terahertz and Millimeter wave Laboratory, Department of Microtechnology and Nanoscience (MC2), Chalmers University of Technology, SE-412 96 Göteborg, Sweden (e-mail: peter.sobis@chalmers.se).

$\mathrm{N}$. Wadefalk is with the Microwave Electronics Laboratory, MC2, Chalmers University of Technology, SE-41296, Göteborg, Sweden.

J. Stake, is with the Gigahertz Centre, Terahertz and Millimetre wave Laboratory, MC2, Chalmers University of Technology, SE-41296, Göteborg, Sweden.

A. Emrich is with Omnisys Instruments AB, August Barks gata 6B, SE42132 V.F., Sweden.

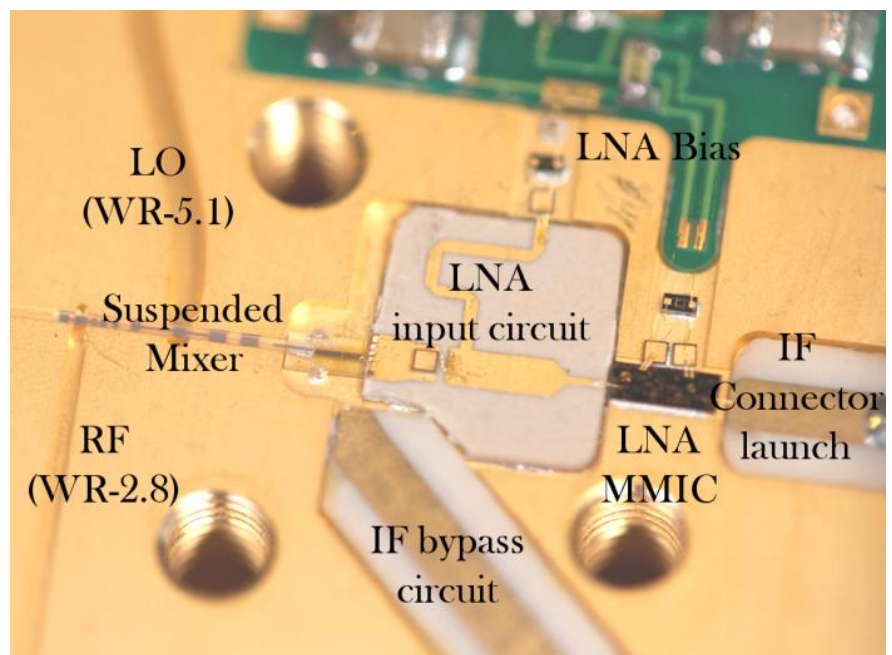

Fig.1. Photo of the receiver split block assembly showing the suspended subharmonic DSB mixer and MMIC IF LNA with accompanying subcircuits.

diode based receiver systems [10-14], typically the mixer and LNA come as two physically separated units interconnected with a $50 \Omega$ interface. This not only increases the IF loss and consequently the receiver noise, but also introduces a strong IF ripple due to the mismatch between the LNA input and the mixer's high IF impedance [15]. Consequently the effective frequency coverage and the accuracy of the instrument are reduced, motivating the integration and co-optimization of the LNA and mixer.

In this paper the advantages of LNA and mixer integration are shown, by a first time demonstration of state-of-the-art performance, in terms of low receiver noise, low ripple and broad instantaneous frequency coverage, of an integrated receiver operating in the $320-360 \mathrm{GHz}$ frequency band. Furthermore, a simple method based on IF characterization of the mixer IF response using a bypass IF circuit and cosimulation of this data with the LNA is presented, allowing for accurate prediction of the receiver noise response and further co-optimization of the mixer and LNA combined response.

\section{DESIGN}

The integrated front-end module, see Fig. 1, was based on a subharmonic Schottky diode mixer, as described in [16] with a 
RF center frequency of around $340 \mathrm{GHz}$, and on a multioctave 4-16 GHz custom MMIC hybrid LNA design developed at Chalmers University of Technology. The LNA had an equivalent minimum input noise temperature ranging from 30 $\mathrm{K}$ to $80 \mathrm{~K}$ with about $30 \mathrm{~dB}$ of gain within the band of operation, see Fig. 2. The mixer used an anti-parallel Schottky diode chip, supplied by Virginia Diodes Inc.. The surface channel planar anti-parallel diodes [17] had a typical pad to pad capacitance of $4 \mathrm{fF}$, a series resistance of $10 \mathrm{Ohm}$, ideality factor of about 1.1 and a total chip capacitance of $10 \mathrm{fF}$. The receiver module housing was of E-plane split block type and measured $24 \mathrm{~mm}$ by $27 \mathrm{~mm}$ by $20 \mathrm{~mm}$ (W x L x H). It accommodated both the RF and LO waveguide interfaces, as well as the mixer, LNA and IF circuits including bias circuitry and the IF connector launch. Thereto a parallel IF channel was also included in the block, allowing for separate testing of the mixer conversion loss, noise and IF impedance, by simply rerouting a wirebond connection.

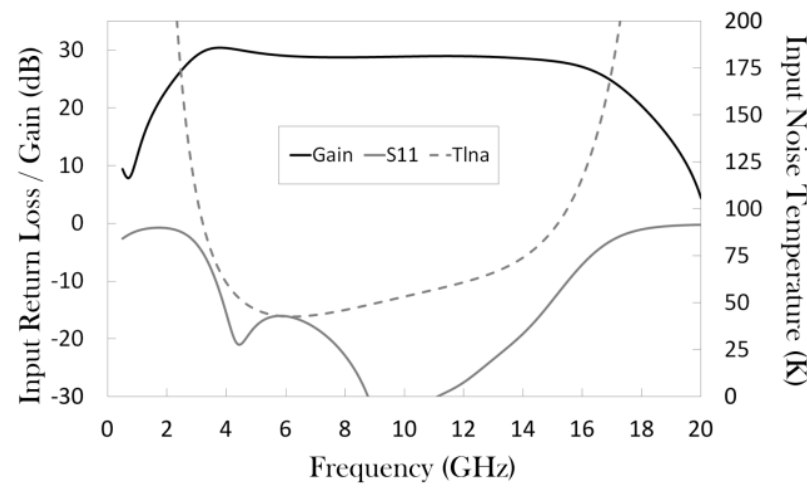

Fig. 2. Gain, input return loss and input noise data of the LNA (50 $\Omega)$.

\section{MeAsurement SetuP}

Two receiver modules were assembled and tested in a semiautomated hot/cold measurement setup described in [16]. A corrugated WR-2.8 horn from Radiometer Physics GmbH was used together with a broadband active LO multiplier source module consisting of an active W-band x6 multiplier from Omnisys and a broadband $170 \mathrm{GHz}$ Schottky varactor doubler from Chalmers, see Fig. 3.

\section{RESULTS AND DISCUSSION}

Both receivers showed an optimum noise performance at an LO power of around $1.2 \mathrm{~mW}$, which was measured by connecting an Erickson PM4 power meter directly to the doubler LO flange.

By the use of an IF bypass circuit, the mixer noise and conversion loss could be estimated. This was done by using a $3 \mathrm{~dB}$ attenuator and a coaxially packaged LNA module based on the same design that was incorporated to the integrated receiver. The complex reflection coefficient of the mixer IF port was characterized up to $20 \mathrm{GHz}$ using a VNA, allowing for detailed modeling of the mixer diode IF impedance and circuit assembly. The mixer conversion loss and noise was estimated in the 6-8 GHz IF range, at which the mixer IF port impedance was close to $50 \mathrm{Ohm}$ and effect of IF ripple

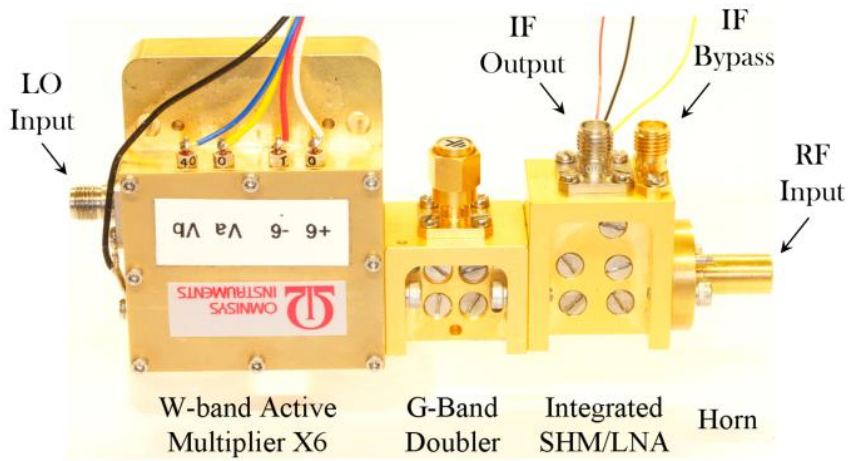

Fig. 3. Photo of the front end receiver module including the LO multiplier chain and corrugated horn as used in the test setup.

minimized, to approximately $6 \mathrm{~dB}$ (DSB) and $780 \mathrm{~K}$ (DSB) respectively. For the integrated receiver the estimated mixer noise at the receiver noise minimum point at around $3.5 \mathrm{GHz}$ $\mathrm{IF}$, using $\mathrm{T}_{\min }$ equal to $33 \mathrm{~K}$ for the LNA input noise and $9 \mathrm{~dB}$ for the mixer conversion loss, was found to be about $760 \mathrm{~K}$. The diode IF impedance was found to be around $200 \Omega$ at an $1.2 \mathrm{~mW}$ LO drive, varying from $130 \Omega$ at high drive to $270 \Omega$ at low drive.

Based on the mixer characterization together with a detailed LNA model and detailed 3D-EM modeling of the packaged mixer circuit IF response, the full receiver noise response can be simulated. The receiver noise was found to strongly depend on the mismatch to the mixer high IF impedance and distance of the LNA to the mixer diode, increasing the noise level and ripple amplitude and the ripple periodicity respectively.

The measured receiver noise of two receiver modules is presented in Fig. 4 together with simulations of the minimum receiver noise based on the minimum input noise temperature $\mathrm{T}_{\min }$ of the LNA, and of the receiver noise based on the performance of the coaxial LNA module, assuming the mixer IF port impedance to be $50 \Omega$. The two modules have very similar responses showing good repeatability. A clear reduction in bandwidth with a slow and small ripple is noticed. Also a noticeable increase of the receiver noise in the

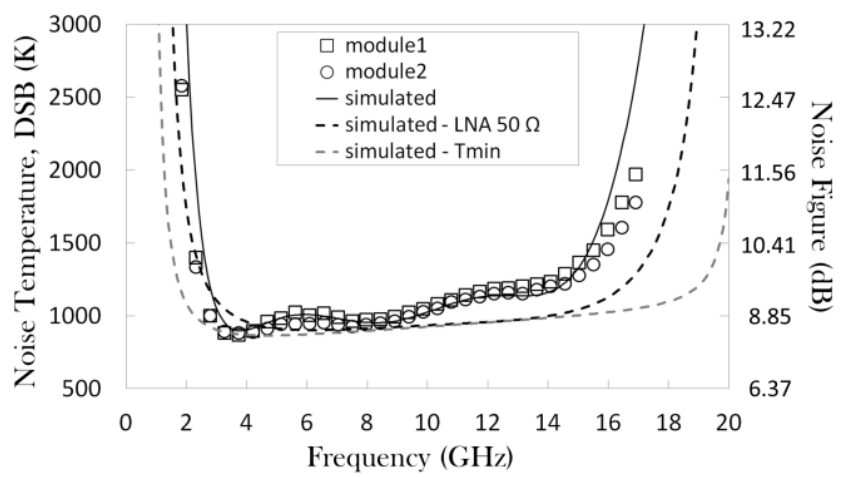

Fig.4. Measured receiver DSB noise temperature versus IF frequency for two assembled integrated receiver modules compared with simulations of the receiver noise taking the mixer and LNA interaction into account as well as estimates of the minimum receiver noise assuming a $50 \Omega$ mixer IF impedance in the first case and $\mathrm{T}_{\min }$ for the LNA for the second case. The mixer conversion loss and noise are assumed to be constant. 


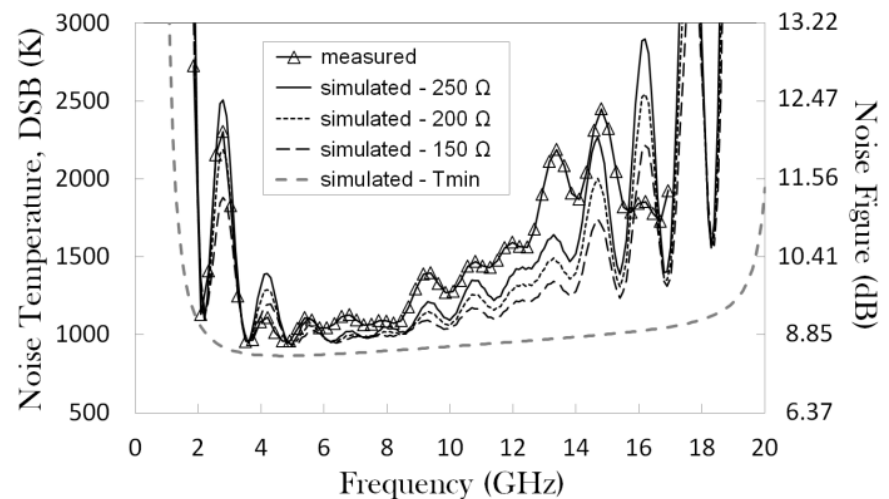

Fig.5. Measured and simulated receiver DSB noise temperature versus IF frequency using an external LNA together with simulations of the receiver noise for different mixer IF impedances.

higher end of the band is shown compared to the case the LNA and mixers IF port had been matched to $50 \Omega$. The ripple minima in the lower part of the IF band also coincide well with the ideal receiver noise response assuming the minimum noise temperature $T_{\min }$ for the LNA and matched conditions.

A constant mixer conversion loss of $6 \mathrm{~dB}$ (DSB), a mixer noise of $770 \mathrm{~K}$ (DSB) and a mixer IF impedance of $200 \Omega$ was used in the simulations. As a fixed LO frequency is used, close to the design center frequency, and due to the relative broad $\mathrm{RF}$ frequency coverage of the mixer, a relatively small variation in the mixer DSB conversion loss and DSB noise could be expected. In Fig. 5 measurements and simulations of the receiver using the external LNA are plotted, for different diode impedances. The main sources for deviations between measurements and simulations are thought to come from the assembly, mainly from mismatches in the coaxial connector interfaces of the LNA and mixer packages, which were not included in the simulations.

\section{CONCLUSION}

A compact receiver front-end module with ultra broadband and flat noise characteristics has been demonstrated, showing the advantage of integrating the mixer and LNA in the same package. By co-simulation of the LNA and mixer IF circuit, using a constant mixer noise, conversion loss and diode IF impedance based on measured results, a good prediction of the receiver noise characteristic was achieved. The noise response of the integrated receiver was found to be largely affected by the mixer IF impedance in combination with the mixer and LNA interaction. Thus for instruments requiring optimum noise performance over large instantaneous IF bandwidths, cooptimization of the mixer and LNA noise combined response is found to be necessary.

\section{ACKNOWLEDGMENT}

The authors would like to acknowledge Associate Professor S. Cherednichenko for helpful discussions and Mr. R. Dahlbäck for assistance in setting up the automated measurement setup, Mr. C.-M. Kihlman for the excellent work of machining the waveguide split block modules, Mr. V. Drakinskiy for help in processing the mixer quartz circuits and
Mr. M. Petersson for assembling the receiver IF circuits. We would also like to acknowledge VDI for supplying diodes for this experiment and Dr. T. Crowe and Dr. G. Schoenthal for helpful discussions.

\section{REFERENCES}

[1] P. Siegel, "THz Instruments for Space," IEEE Transactions on Antennas and Propagation, vol.55, no.11, pp.2957-2965, Nov. 2007.

[2] ESA SP-1313/5 Candidate Earth Explorer Core Missions - Reports for Assessment: PREMIER - Process Exploration through Measurements of Infrared and millimetre-wave Emitted Radiation, 2008, ISBN 978-929221-406-7, ISSN 0379-6566M.

[3] D. Doyle, G. Pilbratt, J. Tauber, "The Herschel and Planck Space Telescopes," Proceedings of the IEEE, vol.97, no.8, pp.1403-1411, Aug. 2009.

[4] M. Cohn, J Degenford, B. Newman, "Harmonic Mixing with an AntiParallel Diode Pair," 1974 S-MTT International Microwave Symposium Digest, vol.74, no.1, pp. 171- 172, Jun 1974.

[5] M. Pospieszalski, "Extremely low-noise amplification with cryogenic FETs and HFETs: 1970-2004," IEEE Microwave Magazine, vol.6, no.3, pp. 62- 75, Sept. 2005.

[6] F. Rodriguez-Morales, K. Yngvesson, Gu Dazhen, "Wideband IFIntegrated Terahertz HEB Mixers: Modeling and Characterization," IEEE Transactions on Microwave Theory and Techniques, vol.58, no.5, pp.1140-1150, May 2010.

[7] B. Thomas, B. Alderman, D. Matheson, P. de Maagt, "A Combined 380 GHz Mixer/Doubler Circuit Based on Planar Schottky Diodes," IEEE Microwave and Wireless Components Letters, vol.18, no.5, pp.353-355, May 2008.

[8] M. Abbasi, S.E. Gunnarsson,; N. Wadefalk, R. Kozhuharov, J. Svedin, S. Cherednichenko, I. Angelov, I. Kallfass, A. Leuther, H. Zirath, "Single-Chip 220-GHz Active Heterodyne Receiver and Transmitter MMICs With On-Chip Integrated Antenna," IEEE Transactions on Microwave Theory and Techniques, vol.59, no.2, pp.466-478, Feb. 2011.

[9] B. Thomas, A. Maestrini, J. Gill, C. Lee, R. Lin, I. Mehdi and P. de Maagt, "A Broadband 835-900-GHz Fundamental Balanced Mixer Based on Monolithic GaAs Membrane Schottky Diodes," IEEE Transactions on Microwave Theory and Techniques, vol. 58, no. 7, Jul 2010.

[10] I. Mehdi, S. Marazita, D. Humphrey, L. Trong-Huang, R. Dengler, J. Oswald, A. Pease, S. Martin, W. Bishop, T. Crowe and P. Siegel, "Improved 240-GHz subharmonically pumped planar Schottky diode mixers for space-borne applications," IEEE Transactions on Microwave Theory and Techniques, vol.46, no.12, pp.2036-2042, Dec 1998.

[11] J. Hesler, W. Hall, T. Crowe, R. Weikle, II, B. Deaver, Jr., R. Bradley, and S. K. Pan, "Fixed-tuned submillimeter wavelength waveguide mixers using planar Schottky barrier diodes," IEEE Trans. Microw. Theory Tech., vol. 45, no. 5, pp. 653-658, May 1997.

[12] T. Crowe, D. Porterfield, J. Hesler, W. Bishop, D. Kurtz and K. Hui, "Terahertz sources and detectors", Proc. SPIE 5790, 271, 2005.

[13] A. Maestrini, B. Thomas, H. Wang C. Jung, J. Treuttel, Y. Jin, G. Chattopadhyay, I. Mehdi and G. Beaudin, "Schottky diode-based terahertz frequency multipliers and mixers," C. R. Physique, vol.11, no.7-8, pp.480-495, 2010.

[14] H.-W. Hubers, "Terahertz Heterodyne Receivers," IEEE Journal of Selected Topics in Quantum Electronics, vol.14, no.2, pp.378-391, March-april 2008.

[15] J. Hesler, "Planar Schottky Diodes in Submillimeter-Wavelength Waveguide Receivers," Ph. D. dissertation, School of Engineering and Applied Science, University of Virginia, Charlottesville, United States 1996.

[16] P. Sobis, A. Emrich and J. Stake, "A Low VSWR 2SB Schottky Receiver," IEEE Trans. on Terahertz Science and Technology, vol.1, no.2, pp.403-411, Nov, 2011.

[17] W. Bishop, K. McKinney, R. Mattauch, T. Crowe, G. Green, "A Novel Whiskerless Schottky Diode for Millimeter and Submillimeter Wave Application," 1987 IEEE MTT-S International Microwave Symposium Digest, vol.2, no., pp.607-610, May 9 1975-June 111987. 